\title{
Spatial orientation of the ventricular muscle band: Physiologic contribution and surgical implications
}

\author{
Francisco Torrent-Guasp, MD, ${ }^{a}$ Manel Ballester, MD, ${ }^{b}$ Gerald D. Buckberg, MD, ${ }^{c}$ Francesc Carreras, MD, ${ }^{d}$ \\ Albert Flotats, MD, ${ }^{\mathrm{d}}$ Ignasi Carrió, MD, ${ }^{\mathrm{d}}$ Ana Ferreira, MD, ${ }^{\mathrm{b}}$ Louis E. Samuels, MD, ${ }^{\mathrm{e}}$ and Jagat Narula, MD, \\ Dènia, Lleida, and Barcelona, Spain; Los Angeles, Calif; and Philadelphia, $\mathrm{Pa}$
}

$\mathrm{I}$ t has been proposed that the ventricular myocardium, both right (RV) and left (LV), exists as a continuous muscle band. ${ }^{1-4}$ The band is oriented spatially as a helix formed by basal and apical loops. We hypothesize that this unique anatomy and spatial configuration of the myocardial muscle determine the way the ventricular ejection and filling take place. ${ }^{5,6}$ Further, knowledge of this unique morphologic and physiologic characteristic should facilitate development of more effective surgical procedures in congestive heart failure.

\section{Unwrapping of the Ventricular Myocardial Band}

Careful anatomic studies have established the way the cardiac band should be unrolled. ${ }^{3,4}$ Unwrapping occurs easily (Figure 1), with least resistance, along a natural cleavage plane. Dissection of the ventricular myocardial band can be accomplished in three steps. In the first step (Figure 2, A), the basal loop is unrolled. The superficial fibers of the anterior aspect of the left ventricle are cut along the anterior interventricular sulcus (see arrow) to pull apart the RV free wall (Figure 2, B). Dissection can then proceed posteriorly following the cleavage plane. In this way the complete basal loop is extended in its full length (Figure 2, C, black and dark gray areas). In the second step (Figure 2, $C$ and $D$ ), the aorta is dismounted, which involves separation along the cleavage plane (see arrow in Figure 2, $C$ ) defined by the two muscular strata, the fibers of the descending segment (white) and the fibers of the ascending

From Dènia, Spain ${ }^{\mathrm{a}}$; Hospital Universitari Arnau de Vilanova and Facultat de Medicina de la Universitat de Lleida, Spain ${ }^{\text {b; }}$ Department of Cardiac Surgery, University of California, Los Angeles, Calif ; ; Hospital de la Santa Crei i Sant Pau, Barcelona, Spain ${ }^{\mathrm{d}}$; and Hahnemann University Hospital, Philadelphia, $\mathrm{Pa}{ }^{\mathrm{e}}$

Received for publication Dec 12, 2000; accepted for publication Dec 15, 2000.

Address for reprints: Manel Ballester, MD, University of Lleida, Department of Cardiology, Alcalde Rovira Roure \#80, 25198 Lleida, Spain (E-mail: mballesterr@mx4.revestb.es)

J Thorac Cardiovasc Surg 2001;122:389-92

Copyright $\odot 2001$ by The American Association for Thoracic Surgery $0022-5223 / 2001 \$ 35.00+0 \quad \mathbf{1 2 / 5 4 / 1 1 3 7 4 5}$

doi:10.1067/mtc.2001.113745

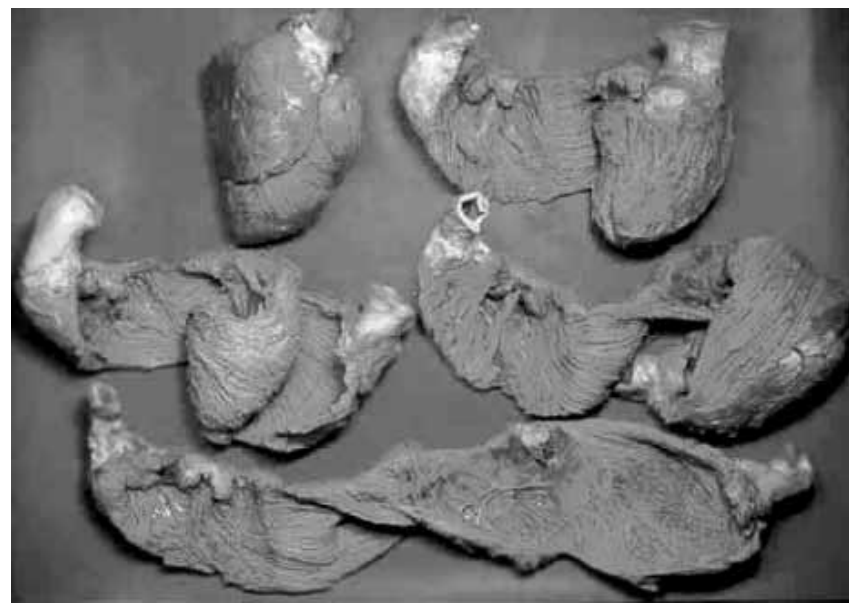

Figure 1. The consecutive stages of the unwinding of the ventricular myocardial band are shown. See Figure 2 (steps A-E) for schematic representation.

segment (light gray). The fibers of ascending and descending segments cross each other at a $90^{\circ}$ angle at the level of the septum. Following such a plane upward to the aorta, right and left trigones are found (see $l t$ and $r t$ in Figure 2,D). After sectioning of both trigones, the aorta can easily be dismounted. In the third step (Figure 2, $D$ and $E$ ), the apical loop is unrolled. Pulling apart the dismounted aorta allows the apical loop to be unrolled so that the entire ventricular myocardial band can be stretched out (Figure 2, $E$ ). Accordingly, the band extends from the pulmonary artery to the aorta (Figures 1 and 2).

\section{Segmental Anatomy of the Band}

At the center of the uncoiled myocardial band, a fold can be observed that twists the band by $180^{\circ}$ and demarcates its basal and apical loops (Figure 2,E). The basal loop runs from the pulmonary artery root $(a)$ to the central fold $(b)$, and the apical loop runs from the central fold $(b)$ to the aortic root $(c)$. Both loops, in turn, can be divided into two segments each. The basal loop is divided into the RV free wall segment ( $R F W$, black), which extends from the pulmonary artery root $(a)$ to the posterior interventricular septum (dotted line $d$ ), and the LV free wall segment ( $L F W$, dark gray), which extends from the posterior interventricular sulcus $(d)$ to the central fold $(b)$. Similarly, the apical 


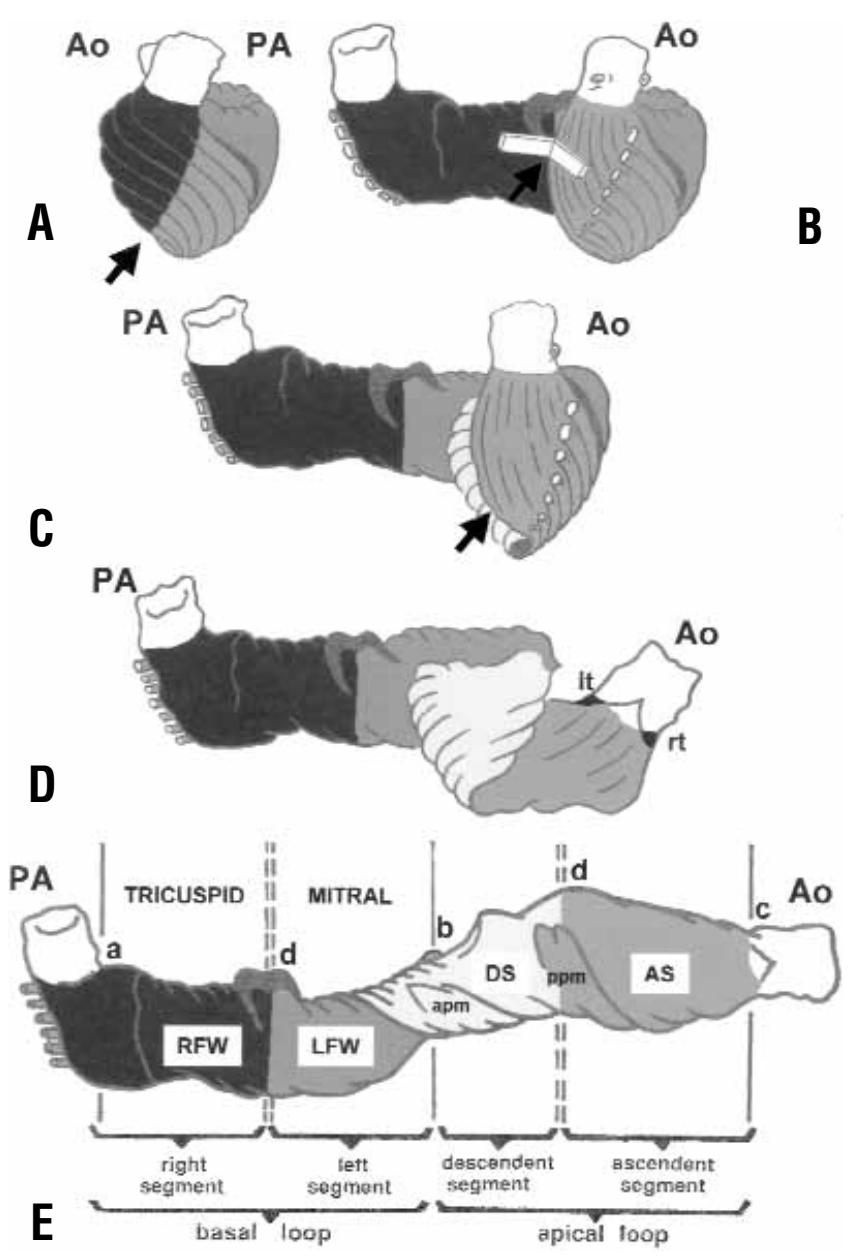

Figure 2. Schematic representation of the ventricular myocardial band $(A-E)$ for consecutive stages of unwinding. The myocardial band can be divided into four segments (E): RV free wall (RFW, black), LV free wall (LFW, dark gray), descending segment (DS, white), and ascending segment (AS, light gray). The myocardial band extends between the pulmonary artery (PA) and the aorta (Ao). These segments spatially conform to two loops: the basal loop (from a to $b$ ), constituted by RFW (black) and LFW (dark gray), and the apical loop (from $b$ to $c$ ) formed by the descending segment (DS, white) and the ascending segment (AS, light gray). The spatial configuration of both loops forms a helicoid as shown in Figure 3. Arrows indicate the cleavage plane that provide clues for unwinding the myocardial band. apm, Anterior papillary muscle; $A S$, ascending segment; $A o$, aorta; $D S$, descending segment; $L F W$, left ventricular free wall; It, left trigone of the aorta; $P A$, pulmonary artery; ppm, posterior papillary muscle; $R F W$, right ventricular free wall; rt, right trigone of the aorta.

loop is also divided into two segments, the descending LV segment ( $D S$, white), which extends from the central fold $(b)$ to the posterior papillary muscle ( $p p m$, see $d$ ), which topographically coincides with the posterior interventricular sulcus when the band is wrapped), and the ascending segment ( $A S$, light gray), which extends from the posterior papillary muscle (ppm) to the aortic root $(c)$. Therefore, the ventricular myocardial band pro-

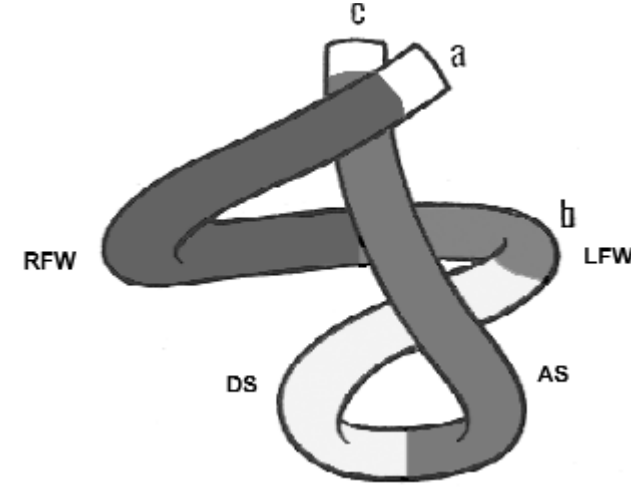

Figure 3. Schematic representation of the spatial configuration of the ventricular myocardial band as a helicoid. Abbreviations and shading as in Figure 2.

duces two helicoid spirals. One spiral is represented by the apical loop and the other by the basal loop (Figure 3).

Conceptualizing Mechanics of Ventricular Contraction Based on Spatial Orientation of Myocardial Band

Anatomy dictates function, and it is likely that the helical anatomic configuration may contribute to ventricular performance. ${ }^{5,6}$ In this respect, analysis of the movements of the heart in cine-loop nuclear magnetic resonance studies of normal individuals ${ }^{7}$ demonstrates lack of movement of the apex during the cardiac cycle. Instead, the entire base of the heart (atria and great vessels) moves downward in systole and upward in diastole. Recently, using fast Fourier analysis of gated blood pool ventriculography, we have observed that the sequence of mechanical ventricular activation closely follows the helicoid trajectory of the myocardial band; the initial segment to be activated is the basal region of the ventricle, first the RV segment and then the LV, and the apical segments follow. ${ }^{8}$ It is conceivable that sequential contractile activity of the peculiar helicoid structure of the ventricular myocardium allows upward and downward movements. The sequence of contraction is as follows: RV free wall $\rightarrow \mathrm{LV}$ free wall $\rightarrow$ descending segment $\rightarrow$ ascending segment. Accordingly, after contraction of the RV free wall segment (black), contraction of the LV free wall segment (dark gray) takes place. As a result, the entire basal loop (both black and dark gray segments) becomes a rigid external cylinder, analogous to the stiff outer shell previously described by Armour and Randall. ${ }^{9}$ Subsequent contraction of the descending segment pulls the ventricular base downward, thereby shortening the long axis of the ventricular cavity, reducing its volume and allowing ventricular ejection. Contraction of the ascending segment then results in an increase in the longitudinal axis of the ventricles and an upward displacement of the base of the heart, which increases the ventricular volume. The increase in volume is similar to recent nuclear magnetic resonance study ${ }^{7}$ and allows for the suction function (or diastole) of the left ventricle, as previously hypothesized. ${ }^{10}$

Therefore, the mechanics of the ventricles resemble those of a cylinder and piston of a motor engine. The cylinder is constituted by the basal loop, the contraction of which forms an outer stiff shell that is pulled down by the contraction of the descending segment and upward by the ascending segment. ${ }^{5}$ Whereas the cylinder is fixed and the piston provides the mobile element in the former, 
the cylinder moves over the fixed piston in the human ventricle.

One intriguing aspect of the ventricular mechanics is the nature of the upward movement of the ventricular base, which elongates the long axis of the ventricular cavities. In nuclear magnetic resonance studies, the basal myocardium moves abruptly upward in the late phase of systole. ${ }^{11}$ The question of why the contraction of the ascending segment should give rise to an increase in the longitudinal axis of the ventricles is of major interest. One way to explain such a movement is proposed in Figure 4. At the time of contraction of the descending segment, which pulls down the entire basal loop (cylinder) of the ventricles, the ascending segment adopts a forced "S" configuration (Figure 4,C). On the other hand, the contraction of the ascending segment (thicker fibers in Figure 4, D) implies its stiffening, which gives rise to lengthening of the long axis of the ventricular cavities. This movement is similar to the way the contraction of the dorsal musculature of the snake is associated with lengthening and upward movement of its body structure (Figure 4, $C, D$, and $E$ ). In the heart, increase of the long axis of the ventricle would give rise to the suction of blood (early rapid filling phase). ${ }^{12}$ Due to the spatial configuration of the ascending and descending segments, a rotational movement of the heart occurs. This confirms the echocardiographic observations of the motion of the papillary muscle ${ }^{3}$ and recent characterization by nuclear magnetic resonance. ${ }^{11}$ In the latter study, a counterclockwise LV twist of the basal portion of the ventricle was detected during isovolumic contraction (probably caused by contraction of the descending segment), whereas the base rotated clockwise during late systole (contraction of the ascending segment). The segmental direction of fibers of both segments shown in Figure 4 explains the rotational movements.

\section{Surgical Implications}

We reason that sequential contraction of the ventricular muscle band, spatially distributed as a helicoid, results in successive shortening and lengthening of the ventricles. These movements may determine the ejection and suction of blood, respectively. Such a concept proposes early diastole as an active process. ${ }^{10}$ It should also alter our approach to the surgical intervention in heart failure. ${ }^{12}$ Reduction ventriculectomy has been recently proposed as a surgical alternative in end-stage heart failure in transplant noncandidates. Although theoretically logical, the technique has not proven surgically efficacious. It is likely that ligation of apical and basal segment muscular planes after partial volume reduction interferes with the natural sequence of myocardial contraction and offsets the benefit accrued from diastolic volume attenuation. It behooves us to develop surgical strategies that precisely shorten the nonoverlapping regions of the ventricular muscle band.

\section{References}

1. Torrent-Guasp F. Anatomía funcional del corazón. Madrid: PazMontalbo, 1957.

2. Torrent-Guasp F. An experimental approach in heart dynamics physiology and pharmacology. Eugene Talmadge Hospital. Augusta (GA). Madrid: S. Aguirre Torre, 1959

3. Streeter DD, Torrent-Guasp F. Geodesic paths in the left ventricle of the mammalian heart. Circulation. 1973;48:471-7.

4. Streeter DD, Powers WE, Ross MA, Torrent-Guasp F. Three-dimensional fiber orientation in the mammalian left ventricular wall. In:
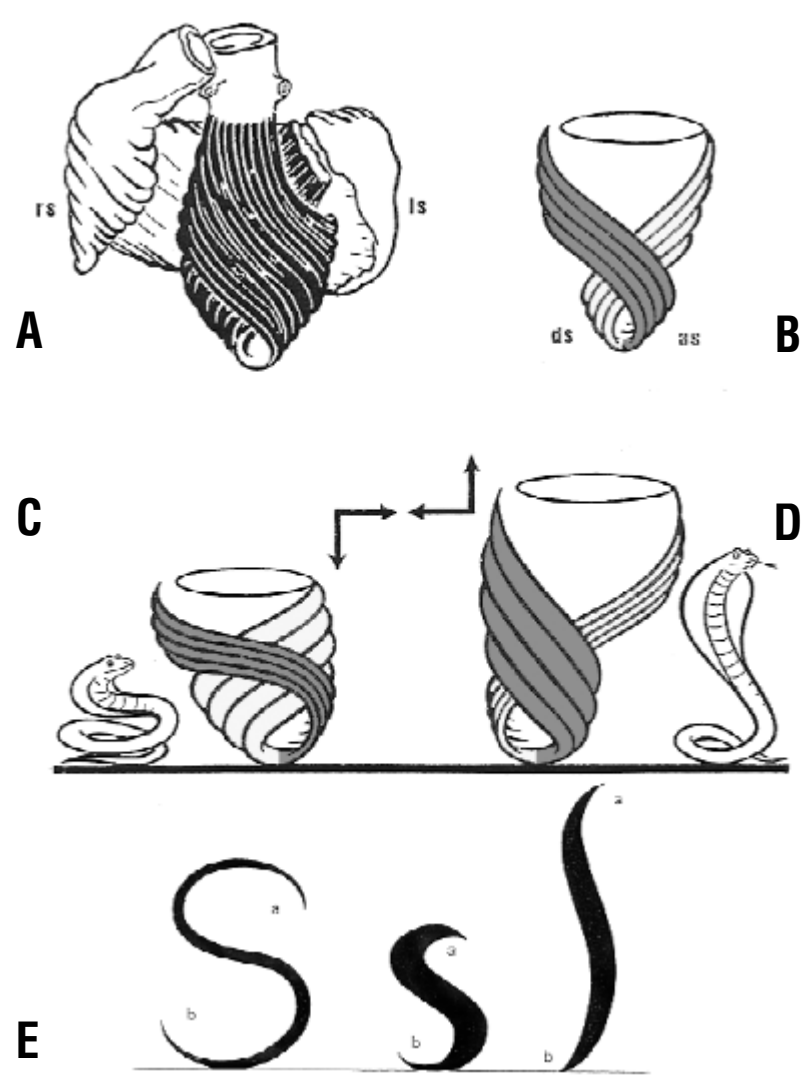

Figure 4. Proposed explanation of the late systolic upward motion of the base of the ventricles. A, The outer shell formed by the basal loop has been unwound to display the descending and ascending segments of the band, which are seen shaded in B. C and D illustrate the way the descending and ascending segments behave during systole. In early systole the base of the heart is pulled toward the apex because of contraction of the descending segment (thick bundles shaded lightly); such a movement forces the ascending segment to adopt an " $S$ " configuration in late systole. Contraction of the ascending segment (thick bundles in light gray) stiffens such a segment and results in upward movement of the base of the heart. The latter movement could be compared to the way contraction of the dorsal musculature of the snake elongates its body through stiffening of the muscles. Depicted in $E$ is a scheme of the forced " $S$ " configuration of the ascending segment (left), the contraction leads to a shortened "s" (middle), and the likely configuration it adopts in late systole (right). Contraction of the descending and ascending fibers also results in a rotational motion of the heart, as illustrated in the two intermediate figures, which depict such movement as seen from the apex.

Barn J, Noordegraf A, Raines J, editors. Cardiovascular system dynamics. Cambridge: MIT Press; 1978. p. 73.

5. Torrent-Guasp F. Estructura y función del corazón. Rev Esp Cardiol. 1998;51:91-102.

6. Torrent-Guasp F, Ballester M, Samuels L, Narula J. Spatial orientation of the ventricular muscle band and approach to partial ventriculotomy in heart failure. In: Narula J, Ballester M, Carrió I, Virmani R, Willerson J, editors. Pathogenetic basis of myocardial disorders. London: ISIS Publishers. In press. 
7. Karwatowski SP, Brecker JD, Yang GZ, Firmin DB, St John-Sutton M, Underwood SR. A comparison of left ventricular myocardial velocity in diastole measured by magnetic resonance and left ventricular filling measured by echocardiography. Eur Heart J. 1996;17: 795-802.

8. Flotats A, Torrent-Guasp F, Ballester-Rodés M, Carrió I, Estorch M, Ballester-Alomar M, et al. Fourier analysis based display of the onset of myocardial contraction: correlation with the sequential contraction in the continuous ventricular myocardial band. J Nucl Cardiol. In press.
9. Armour JA, Randall WC. Electrical and mechanical activity of the papillary muscles. Am J Physiol. 1970;218:1710-7.

10. Brutssaert DL, Sys SU, Gillebert TC. Diastolic failure: pathophysiology and therapeutic implications. J Am Coll Cardiol. 1993;22:318-25.

11. Lorenz CH, Pastorek JS, Bundy JM. Delineation of normal left ventricular twist throughout systole by tagged cine magnetic resonance imaging. J Cardiovasc Magn Res. 2000;2:97-108.

12. Torrent-Guasp F, Caralps-Riera JM, Ballester M. Cuatro propuestas para la remodelación ventricular en el tratamiento quirúrgico de la miocardiopatía dilatada. Rev Esp Cardiol. 1997;50:682-8. 